\title{
On ablation of large Tungsten dust grains in edge plasma of fusion devices
}

\author{
S. I. Krasheninnikov ${ }^{1}$ and E. D. Marenkov ${ }^{2}$ \\ ${ }^{1}$ University California San Diego, La Jolla, CA 92093, USA \\ ${ }^{2}$ Nuclear Research National University MEPhI, Moscow, 115409, Russia
}

\begin{abstract}
The model describing vapor induced shielding effects for $\mathrm{W}$ dust grain evaporation in the edge plasmas is considered. It is shown that unlike the case of pellet ablation models, where it is assumed that the heat flux to pellet is due to free streaming electrons, the heat flux to $\mathrm{W}$ grain in edge plasma is due to electron heat conduction. By using some reasonable simplifications, tractable equations for the evaporation rate of the grain are obtained. Approximate analytical analysis of these equations, supported by numerical solutions, shows that the vapor induced screening effects can reduce the evaporation rate of the grain in edge plasmas by an order of magnitude. This model can also be applied for other high-Z grain materials (e.g. Mo).
\end{abstract}


PACS: 52.40.Hf, 52.55.Fa, 52.27.Lw

JNM keywords: Theory and Modeling

PSI-20 keywords: edge plasma, dust, impurity

*Corresponding author address: UCSD, 9500 Gilman Dr., La Jolla, CA 92093-0411, USA.

*Corresponding author E-mail: skrash@mae.ucsd.edu

Presenting author: S. I. Krasheninnikov

Presenting author E-mail: skrash@mae.ucsd.edu 


\section{Introduction}

The interactions of fusion plasmas with dust grains results in dust charging, acceleration, heating, and ablation of grain material, which results in the pollution of fusion plasma with impurities [1]. If ablation process is rather slow, dust can penetrate through the edge plasma fairly deeply toward the core and, as a result, might cause a lethal pollution of the core plasma. However, in the models of grain-plasma interactions implemented into DUSTT code [1], it is assumed that ablated material of the grain does not alter grain-plasma interactions. This approach is fully justified for relatively small grain effective radius $\mathrm{R}_{\mathrm{d}} \tilde{<} \mathrm{R}_{\mathrm{d}}^{\max } \sim 10 \mu \mathrm{m}$ [2], but is invalid for larger grains. Meanwhile, large grains containing considerable amount of medium- and high-Z materials (e.g. Iron, Tungsten) can impose a serious threat for the performance of fusion plasma (e.g. see Ref. 3). In this work we consider an impact of the vapor induced shielding on ablation of relatively large grains, $R_{d}>R_{d}^{\max }$, made of high-Z material. To be more specific, we will target Tungsten (W) dust as the most probable high-Z dust grain material in ITER. In Section II we comment on applicability of the models, developed for hydrogen pellet ablation to description of $\mathrm{W}$ grain evaporation. In Section III we present our own model and estimate shielding effect for large W gains. In Section IV we summarize our main conclusions.

\section{Assessment of the applicability of pellet ablation models for $W$ dust grain} evaporation in edge plasmas

The key ingredient of all models describing the vapor induced shielding effects for both hydrogen and impurity pellet ablation (e.g. see Ref. 4-11) is calculation of energy loss of ambient plasma electrons (primary electrons) impinging on the vapor/plasma shield of ablated material surrounding the pellet. This energy loss is due to both ionization and excitation of neutrals/ions of the shield, as well as Coulomb collisions with secondary 
electrons produced by the ionization. Elastic collisions of primary electrons with neutrals/ions of the shield divert primary electrons from the pellet and also reduce the energy flux to the pellet. Secondary electrons just after ionization process have a wide energy distribution ranging from zero to the energy of primary electrons minus the ionization potential [12]. If the energy of primary electrons is large enough, secondary ones can continue further ionization/excitation of neutrals/ions of the shield, which results in the formation of secondary plasma around the pellet. This secondary plasma plays an important role in the dissipation of the heat flux to the pellet (e.g. see Ref. 4, 10). The partition between ionization and excitation/radiation channels of electron energy loss depends on the electron density and radiation trapping effects (e.g. see Ref. 12). For relatively large electron and/or neutral densities, when multi-step ionization processes become important [12], the ionization channel dominates.

The primary electron energy loss in neutral vapor part of the shield is assumed to be determined by function $\mathrm{L}_{\mathrm{pe}}(\mathrm{E})$ (where $\mathrm{E}$ is the electron energy), defined as $\mathrm{dE} / \mathrm{dx}=-\mathrm{N}_{\mathrm{a}} \mathrm{L}_{\mathrm{pe}}(\mathrm{E}), \quad$ where $x$ is the distance traveled by an electron and $\mathrm{N}_{\mathrm{a}}$ is the density of vapor atoms. Different approximations are used for $\mathrm{L}_{\mathrm{pe}}(\mathrm{E})$ in the literature. Most of them are expressed in terms of the charge of atom nuclear $Z_{a}$ and intrinsically assume that primary electrons are so energetic, that can dissipate their energy on all electrons of a neutral atom. Obviously, such approximation is invalid for the case of tungsten vapor $\left(Z_{a}=74\right)$ at the edge of fusion devices with electron/ion temperature of ambient plasma $\mathrm{T}_{\infty} \sim 100 \mathrm{eV}$. It was shown that if the sublimation/evaporation energy of vapor material, $\mathrm{E}_{\mathrm{ev}}$ is of the order of several $\mathrm{eV}$, shielding of pellets (or dust grains) with just neutral vapor cloud is rather inefficient (e.g. see Ref. 7-9, 11). This can be understood from the following simple consideration. Let us estimate maximum efficiency of neutral vapor shielding assuming that 
the heat flux is determined by a free-streaming ambient electrons, $\mathrm{q}_{\infty}=\mathrm{n}_{\infty} \mathrm{T}_{\infty}\left(\mathrm{T}_{\infty} / \mathrm{m}\right)^{1 / 2}$ (here $\mathrm{n}_{\infty}$ is the ambient plasma density and $\mathrm{m}$ is electron mass), and it is converted entirely into vaporization/ ablation of the grain. Let us suggest, that the vapor is moving away from the grain with the sound speed $\left(\mathrm{T}_{\mathrm{d}} / \mathrm{M}_{\mathrm{a}}\right)^{1 / 2}\left(\mathrm{~T}_{\mathrm{d}}\right.$ and $\mathrm{M}_{\mathrm{a}}$ are the temperature and atomic mass of evaporated grain material) in radial directions. Then, the "optical depth", $\varsigma_{a}=\int_{0}^{\infty} N_{a}(r) d r$, of the vapor cloud can be estimated as $\varsigma_{\mathrm{a}} \sim 0.25 \mathrm{R}_{\mathrm{d}} \mathrm{n}_{\infty}\left(\mathrm{T}_{\infty} / \mathrm{E}_{\mathrm{ev}}\right)\left(\mathrm{M}_{\mathrm{a}} / \mathrm{m}\right)^{1 / 2}\left(\mathrm{~T}_{\infty} / \mathrm{T}_{\mathrm{d}}\right)^{1 / 2}$, where $R_{d}$ is the grain radius. From electron energy equation we find that the degradation of primary electron energy in the vapor cloud is $\Delta \mathrm{E} / \mathrm{E} \sim \varsigma_{\mathrm{a}} \mathrm{L}_{\mathrm{pe}}\left(\mathrm{T}_{\infty}\right)$. Using expression $\mathrm{L}_{\mathrm{pe}}(\mathrm{E})_{\left[\mathrm{eV} \cdot \mathrm{cm}^{2}\right]}=\mathrm{Z}_{\mathrm{a}} 10^{-11} /\left(4.7 \cdot 10^{3}+8 \cdot \mathrm{E}_{[\mathrm{eV}]}\right) \quad[13]$, for Carbon grain, $\mathrm{T}_{\mathrm{d}} \sim 0.5 \mathrm{eV}$, $\mathrm{R}_{\mathrm{d}} \sim 10^{-2} \mathrm{~cm}$, and $n_{\infty}=3 \cdot 10^{13} \mathrm{~cm}^{-3}$, we find that $\Delta \mathrm{E} / \mathrm{E} \tilde{<} 0.25$ and the vapor shield is, indeed, ineffective. This is in agreement with the conclusions made in Ref. 2, where it was shown that the main impact of plasma vapor on, in particular, tungsten grain-plasma interactions is due to vapor ionization which causes build-up of the plasma shield around the grain.

To assess an impact of shielding effects of secondary plasma, we assume that the ambient plasma parameters remain unchanged and the heat flux to the grain is determined by the free-streaming energy flux of ambient electrons. Then, secondary plasma will build-up within the distance $R_{d}+I_{\text {ion }}$ from the center of the grain, where $I_{\text {ion }}$ is the ionization mean free path of the vapor atoms, simultaneously flowing along the magnetic field lines. For a tungsten dust grain with $\mathrm{R}_{\mathrm{d}} \sim 10^{-2} \mathrm{~cm}$ in ambient plasma with $\mathrm{T}_{\infty} \sim 100 \mathrm{eV}$, and $\mathrm{n}_{\infty} \sim 310^{13} \mathrm{~cm}^{-3}$ we find $\mathrm{I}_{\text {ion }}<\mathrm{R}_{\mathrm{d}}$. Since the cross-field motion of the bump of secondary plasma is due to $\mathbf{E} \times \mathbf{B}$ drift associated with plasma polarization caused by $\operatorname{grad}(\mathbf{B})$ and curvature effects, the extend of secondary plasma along the magnetic field lines crossing the 
grain, $\mathrm{I}_{\|}$, can be estimated [10] as $\mathrm{I}_{\|} \sim \sqrt{\left(\mathrm{R}_{\mathrm{d}}+\mathrm{I}_{\text {ion }}\right) \mathrm{R}} \sim \sqrt{\mathrm{R}_{\mathrm{d}} \mathrm{R}}$, where $\mathrm{R}$ is the tokamak major radius. Then we find the following expression for the ratio $\varsigma_{C}=I_{\|} / \lambda_{C}\left(T_{\infty}, n_{i m p}\right)$, where $\lambda_{C}\left(\mathrm{~T}_{\infty}, \mathrm{n}_{\mathrm{imp}}\right)$ is the Coulomb mean free path of ambient electrons with respect to collisions with impurity ions of secondary plasma,

$$
\varsigma_{\mathrm{C}} \sim \frac{4 \pi \mathrm{e}^{4} \mathrm{Z}^{2} \Lambda \mathrm{n}_{\infty} \sqrt{\mathrm{R}_{\mathrm{d}} \mathrm{R}}}{\mathrm{T}_{\infty} \mathrm{E}_{\mathrm{ev}}}\left(\frac{\mathrm{M}_{\mathrm{a}}}{\mathrm{Zm}_{\mathrm{m}}}\right)^{1 / 2}
$$

where $\mathrm{Z}$ is the charge number of impurity ions in secondary plasma. For tungsten in the plasma with the electron temperature $\mathrm{T}_{\infty} \sim 100 \mathrm{eV}$, the averaged charge calculated within the coronal approximation is $\sim 10$ [14]. As a result, for $\mathrm{R}_{\mathrm{d}} \sim 10^{-2} \mathrm{~cm}, \mathrm{R} \sim 10^{2} \mathrm{~cm}, T_{\infty} \sim 100 \mathrm{eV}$, and $\mathrm{n}_{\infty} \sim 10^{14} \mathrm{~cm}^{-3}$ from Eq. (1) we find $\zeta_{C}=3 \cdot 10^{3}>>1$, which shows an extremely strong attenuation of the electron free streaming flux in the secondary plasma.

\section{Model for self-consistent description of both vapor and secondary plasma effects for W dust grain evaporation in edge plasmas}

Although Eq. (1) overestimates the effect of the grain screening by secondary plasma (recall that we assumed that the electron temperature and the heat flux to the grain are not altered), nonetheless, it clearly demonstrates a crucial role of the secondary plasma. In particular, large value of $\varsigma_{C}$ indicates the change of the heat transfer mechanism to the grain from electron free streaming to conductive.

The physics of large tungsten dust grain interaction with edge plasma, which includes vapor and secondary plasma effects, multiple ionization states, radiation effects, plasma parallel and cross-field dynamics, possible impact of different plasma instabilities, etc. is very complex, so that even available codes [6] cannot account for all these ingredients. Therefore, 
a tractable analytical model describing tungsten dust screening can only be developed by making some significant, but reasonable approximations. In what follows we will assume that: i) The grain is surrounded (see Fig. 1) by partly ionized vapor cloud in the vicinity of the grain, around which is located secondary tungsten plasma. The shape of the vapor cloud is assumed to be close to spherical, with radius $\mathrm{I}_{\mathrm{vc}}>\mathrm{R}_{\mathrm{d}}, \rho_{\mathrm{i}}\left(\rho_{\mathrm{i}}\right.$ is the ion Larmor radius), and its interface with secondary plasma is determined by reaching the ionization degree $\sim 1$; ii) Major shielding impact is, in accordance with our estimates from Sec. II, due to secondary plasma; iii) In relatively high temperature secondary plasma, both ionization state of tungsten ions and radiation loss are described by coronal approximation; iv) The heat flux towards the grain from ambient plasma through secondary plasma and partly ionized vapor cloud is due to electron heat conduction; v) The main mechanism of secondary plasma cross-field transport is due to $\dot{\mathrm{E}} \times \dot{\mathrm{B}}$ drift associated with plasma polarization caused by $\operatorname{grad}(\mathrm{B})$ and curvature effects; vi) Total pressure, $\mathrm{P}_{\text {sh }}$, in both vapor cloud and secondary plasma remains almost constant and is assumed to be much larger than the ambient plasma pressure, $\mathrm{P}_{\mathrm{a}} \sim \mathrm{n}_{\infty} \mathrm{T}_{\infty}$; vii) The flow of high pressure secondary plasma along the magnetic field lines reaches Mach 1 at the interface with ambient plasma; viii) Due to large tungsten atomic mass, $\mathrm{M}_{\mathrm{W}}$, collisional electron-neutral/ion heat transfer is ignored so that temperature of tungsten atoms/ions remains of the order of the temperature of dust material, $T_{d}$; ix) Plasma density in relatively cold and partly ionized vapor cloud is described by Saha equilibrium; $x$ ) Possible impact of radiation from secondary plasma on grain heat load is neglected due to large disparity in sizes of secondary plasma and the grain.

Based on these simplifying assumptions we formulate a tractable set of equations allowing us to account for the vapor induced shielding effects for tungsten grain. We start with energy balance equation in secondary plasma 


$$
\frac{\mathrm{d}}{\mathrm{dl}}\left(\kappa_{\mathrm{e}}(\mathrm{T}) \frac{\mathrm{dT}}{\mathrm{dl}}\right)=\mathrm{n}_{\mathrm{e}} \mathrm{n}_{\mathrm{W}} \mathrm{L}_{\mathrm{W}}(\mathrm{T}) \equiv \frac{\mathrm{P}_{\mathrm{sh}}^{2}}{\mathrm{~T}^{2} \mathrm{Z}_{\mathrm{W}}(\mathrm{T})} \mathrm{L}_{\mathrm{W}}(\mathrm{T}),
$$

where $\mathrm{I}$ is the coordinate along the magnetic field line, $\mathrm{I}=0$ and $\mathrm{I}=\mathrm{I}_{\|}$correspond to the interfaces between secondary plasma and vapor cloud and ambient plasma respectively, $\mathrm{T}$ is the electron temperature, $\mathrm{Z}_{\mathrm{W}}(\mathrm{T})$ is the averaged charge number of tungsten ions, $\mathrm{n}_{\mathrm{W}}$ and $\mathrm{n}_{\mathrm{e}}=\mathrm{Z}_{\mathrm{W}}(\mathrm{T}) \mathrm{n}_{\mathrm{W}}$ are the electron and tungsten ion density, $\kappa_{\mathrm{e}}(\mathrm{T})$ is the electron heat conduction coefficient in fully ionized secondary plasma, $\mathrm{L}_{\mathrm{W}}(\mathrm{T})$ is the cooling function. In Eq. (2) we assumed that the major radiation loss occurs at $Z_{W}>1$. We solve Eq. (2) assuming a strong reduction of electron temperature toward the grain in secondary plasma, so that we can take $\mathrm{T} \sim 0$ at the interface between vapor cloud and secondary plasma. As a result, taking into account the boundary condition $\mathrm{T}\left(\mathrm{I}_{\|}\right)=\mathrm{T}_{\infty}$ we have

$$
\int_{0}^{\mathrm{T}_{\infty}} \frac{\kappa_{\mathrm{e}}(\mathrm{T}) \mathrm{dT}}{\left\{\mathrm{q}_{\mathrm{vc}}^{2}+2 \mathrm{P}_{\mathrm{sh}}^{2} \int_{0}^{\mathrm{T}} \frac{\mathrm{L}_{\mathrm{W}}\left(\mathrm{T}^{\prime}\right) \kappa_{\mathrm{e}}\left(\mathrm{T}^{\prime}\right) \mathrm{dT} \mathrm{T}^{\prime}}{\left(\mathrm{T}^{\prime}\right)^{2} \mathrm{Z}_{\mathrm{W}}\left(\mathrm{T}^{\prime}\right)}\right\}^{1 / 2}}=\mathrm{I}_{\|},
$$

where $\mathrm{q}_{\mathrm{vc}}$ is the heat flux to the vapor cloud. Next we consider the energy transport in partly ionized vapor cloud. We will assume that the optical depth of the cloud is large and ignore all radiation losses from there and use Saha equilibrium for the ionization degree, $\xi_{e}$. Then, from electron heat conduction equation we have

$$
\mathrm{q}_{\mathrm{vc}}=\frac{(\mathrm{T} / \mathrm{m})}{\mathrm{K}_{\mathrm{eW}}(\mathrm{T})} \frac{\mathrm{n}_{\mathrm{e}}}{\mathrm{N}} \frac{\mathrm{dT}}{\mathrm{dl}} \equiv \frac{2\left(\mathrm{~T}_{\mathrm{ed}}^{2} / \mathrm{m}\right)}{\mathrm{K}_{\mathrm{eW}}} \frac{\mathrm{T}_{\mathrm{ed}}}{\mathrm{I}_{\mathrm{W}}} \frac{\mathrm{d} \xi_{\mathrm{e}}}{\mathrm{dl}}
$$


where $\mathrm{I}$ is the coordinate along the magnetic field line, $\mathrm{I}=0$ and $\mathrm{I}=\mathrm{I}_{\mathrm{vc}}$ correspond to the interfaces between the grain and secondary plasma respectively, $\xi_{\mathrm{e}}=\mathrm{n}_{\mathrm{e}} / \mathrm{N} \propto \exp \left(-\mathrm{I}_{\mathrm{W}} / 2 \mathrm{~T}\right)$, $\mathrm{N}$ is the neutral atoms density, $\mathrm{K}_{\mathrm{eW}}(\mathrm{T})$ is the electron-neutral tungsten elastic collision rate constant, $\mathrm{I}_{\mathrm{W}}$ is the tungsten ionization potential, and $\mathrm{T}_{\mathrm{ed}}$ is the electron temperature in the cloud, which varies much slower than the plasma density and, therefore, for simplicity can be taken as constant. Assuming $\xi_{\mathrm{e}} \sim 1$ at the interface between the vapor cloud and secondary plasma and taking into account that the heat flux to the grain is directly related to the total vapor flux from the grain, we have

$$
\mathrm{q}_{\mathrm{vc}}=\mathrm{E}_{\mathrm{ev}} \Gamma / 2 \pi \mathrm{R}_{\mathrm{d}}^{2} \varepsilon\left(\mathrm{T}_{\mathrm{d}}\right)
$$

where $\Gamma$ is the ablation rate (particles/s), $E_{e v}$ is the evaporation energy and $\varepsilon\left(T_{d}\right)$ is the fraction of the heat flux balanced by the evaporation cooling. From Eq. (4) we find

$$
\mathrm{I}_{\mathrm{vc}}=\mathrm{R}_{\mathrm{d}}\left(\Gamma_{0} / \Gamma\right)
$$

where

$$
\Gamma_{0}=4 \pi \varepsilon\left(\mathrm{T}_{\mathrm{d}}\right) \mathrm{R}_{\mathrm{d}} \frac{\mathrm{T}_{\mathrm{ed}} / \mathrm{m}}{\mathrm{K}_{\mathrm{eW}}} \frac{\mathrm{T}_{\mathrm{ed}}^{2}}{\mathrm{E}_{\mathrm{ev}} \mathrm{I}_{\mathrm{W}}}
$$

Since we suppose that $\mathrm{I}_{\mathrm{vc}}>\mathrm{R}_{\mathrm{d}}$, our theoretical consideration is limited to $\Gamma<\Gamma_{0}$. Then, following Ref. 10 and considering cross-field dynamics of secondary plasma caused by $\operatorname{grad}(\mathrm{B})$ and curvature effects and using Eq. (6) we find a relation between $\ell_{\|}$and $\ell_{\mathrm{vc}}$ 


$$
\mathrm{I}_{\|}=\sqrt{\mathrm{I}_{\mathrm{vc}} \mathrm{R}}=\left(\mathrm{R}_{\mathrm{d}} \mathrm{R}\right)^{1 / 2}\left(\Gamma_{0} / \Gamma\right)^{1 / 2},
$$

as well as the expression for $\Gamma$

$$
\left.\Gamma=\frac{\left.2 \pi\right|_{\mathrm{vc}} ^{2} \mathrm{P}_{\mathrm{sh}}}{\left(\mathrm{M}_{\mathrm{W}} \mathrm{T}_{\infty} \mathrm{Z}_{\infty}\right)^{1 / 2}}=\frac{2 \pi \mathrm{R}_{\mathrm{d}}^{2} \mathrm{P}_{\mathrm{sh}}}{\left(\mathrm{M}_{\mathrm{W}} \mathrm{T}_{\infty} \mathrm{Z}_{\infty}\right)^{1 / 2}} \frac{\digamma_{0}}{\Gamma}\right)^{2},
$$

where $\mathrm{Z}_{\infty}=\mathrm{Z}\left(\mathrm{T}_{\infty}\right)$. Combining Eq. $(3,5,8,9)$ we arrive to the equation,

$$
\begin{gathered}
\int_{0}^{\mathrm{T}_{\infty}} \frac{\kappa_{\mathrm{e}}(\mathrm{T}) \mathrm{dT}}{\left\{\left(\frac{\mathrm{E}_{\mathrm{ev}}}{\varepsilon\left(\mathrm{T}_{\mathrm{d}}\right)}\right)^{2}+2\left(\frac{\Gamma}{\Gamma_{0}}\right)^{4}\left(\mathrm{M}_{\mathrm{W}} \mathrm{T}_{\infty} \mathrm{Z}_{\infty}\right) \int_{0}^{\mathrm{T}} \frac{\mathrm{L}_{\mathrm{W}}\left(\mathrm{T}^{\prime}\right) \kappa_{\mathrm{e}}\left(\mathrm{T}^{\prime}\right) \mathrm{dT}^{\prime}}{\left(\mathrm{T}^{\prime}\right)^{2} \mathrm{Z}_{\mathrm{W}}\left(\mathrm{T}^{\prime}\right)}\right\}^{1 / 2}} \\
=\frac{\left(\mathrm{R}_{\mathrm{d}} \mathrm{R}\right)^{1 / 2}}{2 \pi \mathrm{R}_{\mathrm{d}}^{2}} \Gamma_{0}\left(\frac{\Gamma}{\Gamma_{0}}\right)^{1 / 2} .
\end{gathered}
$$

which, along with the expressions

$$
\varepsilon\left(\mathrm{T}_{\mathrm{d}}\right)=\left(1+4 \pi \mathrm{R}_{\mathrm{d}}^{2} \varepsilon_{\mathrm{gr}} \sigma_{\mathrm{SB}} \mathrm{T}_{\mathrm{d}}^{4} / \mathrm{E}_{\mathrm{ev}} \Gamma\right)^{-1}, \Gamma=\pi \mathrm{R}_{\mathrm{d}}^{2} \mathrm{P}_{\mathrm{sat}}\left(\mathrm{T}_{\mathrm{d}}\right) /\left(\mathrm{M}_{\mathrm{W}} \mathrm{T}_{\mathrm{d}}\right)^{1 / 2},
$$

found from the energy balance equation of the grain and the relation between evaporation flux and the saturation vapor pressure, determins the evaporation rate (here $\varepsilon_{\mathrm{gr}}$ is the gray parameter and $\sigma_{\mathrm{SB}}$ is the Stefan-Boltzmann constant). 


\section{Analysis of the model for $W$ dust grain evaporation in edge plasmas}

We start analysis of Eq. $(10,11)$ with simplified analytic estimates and consider some limiting cases. First, we will assume that $\varepsilon\left(\mathrm{T}_{\mathrm{d}}\right) \sim 1$, which means that one can neglect the grain cooling due to light emission. Next we notice that $\mathrm{L}_{\mathrm{W}}(\mathrm{T})$ is virtually constant, $\mathrm{L}_{\mathrm{W}}(\mathrm{T})=\mathrm{L}_{0} \sim 10^{-6} \mathrm{eV} \cdot \mathrm{cm}^{3} / \mathrm{s}$, for $30 \mathrm{eV} \tilde{<} \mathrm{T} \tilde{<} 400 \mathrm{eV}$, which covers entire temperature range of interest in edge plasmas [14]. Thermal conduction coefficient is given by classical equation:

$$
\kappa_{\mathrm{e}}(\mathrm{T})=\frac{16 \sqrt{2}}{\pi^{3 / 2}} \frac{\mathrm{T}^{5 / 2}}{\mathrm{Z}_{\mathrm{W}}(\mathrm{T}) \mathrm{e}^{4} \Lambda \sqrt{\mathrm{m}}}
$$

where $\Lambda$ is the Coulomb logarithm.

Therefore, we can approximate integral expression (10) with the algebraic one:

$$
\frac{\Gamma}{\Gamma_{0}}\left(1+\alpha_{\mathrm{L}}\left(\mathrm{T}_{\infty}\right)\left(\frac{\Gamma}{\Gamma_{0}}\right)^{4}\right)=\left(\frac{\Gamma_{\kappa}\left(\mathrm{T}_{\infty}\right)}{\Gamma_{0}}\right)^{2}
$$

where

$$
\Gamma_{\kappa}\left(\mathrm{T}_{\infty}\right)=\frac{2 \pi \mathrm{R}_{\mathrm{d}}^{2} \kappa_{\mathrm{e}}\left(\mathrm{T}_{\infty}\right) \mathrm{T}_{\infty}}{\mathrm{E}_{\mathrm{ev}}\left(\mathrm{R}_{\mathrm{d}} \mathrm{R}\right)^{1 / 2}} \propto \frac{\mathrm{R}_{\mathrm{d}}^{3 / 2} \mathrm{~T}_{\infty}^{7 / 2}}{\mathrm{Z}_{\infty}}, \quad \alpha_{\mathrm{L}}\left(\mathrm{T}_{\infty}\right)=\frac{\kappa_{\mathrm{e}}\left(\mathrm{T}_{\infty}\right) \mathrm{L}_{0}}{\mathrm{E}_{\mathrm{ev}}} \frac{\mathrm{M}_{\mathrm{W}}}{\mathrm{E}_{\mathrm{ev}}} \propto \frac{\mathrm{T}_{\infty}^{5 / 2}}{\mathrm{Z}_{\infty}}
$$

The second term in the brackets, on the left hand side of Eq. (12), describes the reduction of the heat flux caused by impurity radiation. Since our model is only valid for $\Gamma<\Gamma_{0}$, impurity radiation loss becomes important within our model for $\alpha_{L}\left(T_{\infty}\right) \propto T_{\infty}^{5 / 2} / Z_{\infty}>1$. For $\mathrm{T}_{\infty} \sim 30 \mathrm{eV}, \mathrm{R}_{\mathrm{d}} \sim 10^{-2} \mathrm{~cm}, \mathrm{R} \sim 1.5 \cdot 10^{2} \mathrm{~cm}, \mathrm{~T}_{\mathrm{ed}} \sim 1 \mathrm{eV}$, and $\mathrm{K}_{\mathrm{eW}} \sim 10^{-7} \mathrm{~cm}^{3} / \mathrm{s}$ from Eq. $(7,13)$ we find $\Gamma_{0} \sim 5 \cdot 10^{19} \mathrm{~s}^{-1}, \alpha_{\mathrm{L}}\left(\mathrm{T}_{\infty}\right) \sim 6 \cdot 10^{5}$ and $\Gamma_{\mathrm{K}}\left(\mathrm{T}_{\infty}\right) \sim 10^{19} \mathrm{~s}^{-1}$. For this case, one can see from Eq. (11) that the "radiation" term does not contribute much into the reduction of 
$\Gamma$ and $\Gamma \sim 5 \cdot 10^{18} \mathrm{~s}^{-1}$. We notice that this flux is by order of magnitude smaller then the evaporation flux estimated in [15] for $\mathrm{T}_{\infty} \sim 30 \mathrm{eV}$ and $\mathrm{n}_{\infty} \sim 10^{14} \mathrm{~cm}^{-4}$ with no shielding effects and plasma heat flux to the grain, which is largely controlled by ambient plasma ion flow [1].

For relatively low ambient plasma temperatures, when radiation effects can be ignored, from Eq. (12) we find the following scaling

$$
\Gamma=\Gamma_{\kappa}\left(\mathrm{T}_{\infty}\right) \frac{\Gamma_{\kappa}\left(\mathrm{T}_{\infty}\right)}{\Gamma_{0}} \propto \frac{\mathrm{R}_{\mathrm{d}}^{2} \mathrm{~T}_{\infty}^{7}}{\mathrm{Z}_{\infty}^{2}}
$$

For higher temperatures, when radiation effects become important, we have

$$
\Gamma=\frac{\Gamma_{0}}{\left(\alpha_{\mathrm{L}}\left(\mathrm{T}_{\infty}\right)\right)^{1 / 5}}\left(\frac{\Gamma_{\mathrm{K}}\left(\mathrm{T}_{\infty}\right)}{\Gamma_{0}}\right)^{2 / 5} \propto \frac{\mathrm{R}_{\mathrm{d}}^{6 / 5} \mathrm{~T}_{\infty}^{9 / 10}}{\mathrm{Z}_{\infty}^{1 / 5}}
$$

We now recall that the applicability of heat conduction model of the heat flux to the grain requires large ratio $\varsigma_{C}$ of the characteristic scale length $I_{\|}$to the Coulomb mean free path of electrons in secondary plasma. In addition, we assumed that $\mathrm{I}_{\mathrm{vc}}>\mathrm{R}_{\mathrm{d}}$, which requires $\Gamma_{0}>\Gamma$. After some algebra we find that $\varsigma_{C}>1$ and $I_{v c}>R_{d}$ are valid if the following inequalities are satisfied:

$$
\frac{\Gamma_{\kappa}\left(\mathrm{T}_{\infty}\right)}{\Gamma_{0}} \frac{\mathrm{E}_{\mathrm{ev}}}{\mathrm{T}_{\infty}}\left(\frac{\mathrm{m}}{Z_{\infty} \mathrm{M}_{\mathrm{W}}}\right)^{1 / 2}<\left(\frac{\Gamma}{\Gamma_{0}}\right)^{5 / 2}<1 .
$$


In addition to that, following our assumptions, we should ensure that $\mathrm{P}_{\mathrm{sh}}>\mathrm{P}_{\mathrm{a}}$. Estimating $\mathrm{P}_{\mathrm{sh}}$ from Eq. (9) and again recalling that the relation $I_{v c}>R_{d}$ should hold, we come to the following inequalities

$$
\frac{2 \pi \mathrm{R}_{\mathrm{d}}^{2} \mathrm{n}_{\infty}}{\Gamma_{0}}\left(\frac{\mathrm{T}_{\infty}}{\mathrm{M}_{\mathrm{W}} \mathrm{Z}_{\infty}}\right)^{1 / 2}<\left(\frac{\Gamma}{\Gamma_{0}}\right)^{3}<1
$$

For $\mathrm{T}_{\infty} \sim 30 \mathrm{eV}, \mathrm{n}_{\infty} \sim 10^{14} \mathrm{~cm}^{-3}, \mathrm{R}_{\mathrm{d}} \sim 10^{-2} \mathrm{~cm}$, and $\mathrm{R} \sim 1.5 \cdot 10^{2} \mathrm{~cm}$ inequalities $(16,17)$ hold within a large margin. However, from Eq. (12-14) one finds that they break for both relatively low and high temperatures. At low temperatures, $\mathrm{T}_{\infty} \tilde{<} \mathrm{T}_{\text {low }} \sim 10 \mathrm{eV}$, evaporation flux (14) is not too strong to create significant secondary plasma density. While for rather high temperatures, $\mathrm{T}_{\infty} \tilde{>} \mathrm{T}_{\text {high }} \sim$ few $\times 100 \mathrm{eV}$, our model becomes inapplicable either due to relatively small vapor cloud thickness $\mathrm{I}_{\mathrm{vc}} \sim \mathrm{R}_{\mathrm{d}}$ or due to violation of the heat conduction approximation. We also note that from Eq. (12-14) it is possible to show that the applicability of our model breaks for smaller size grains, showing, in agreement with Ref. 2 that shielding effects are less important for small grains.

In Fig. 2 we show the ablation flux $\Gamma$ as the function of $R_{d}$ for different $T_{i n f}$, found from numerical solution of Eq. $(10,11)$. As we see our analytic estimates are in a reasonable agreement with numerical solutions. More complete numerical results and detailed discussion of applicability limits of our model will be published elsewhere.

\section{Conclusions}

We consider the model describing vapor induced shielding effects for $\mathrm{W}$ dust grain evaporation in the edge of fusion plasma. We demonstrate that unlike the case of pellet ablation models [4-11], where it is assumed that the heat flux to a pellet is due to free 
streaming electrons, the heat flux to a $\mathrm{W}$ grain in the edge plasma is due to electron heat conduction. By using reasonable simplifications we are able to come up with tractable expressions $(10,11)$ for the evaporation rate of the grain. Approximate analytical analysis of these expressions shows that the vapor induced shielding effects can reduce the evaporation rate of the grain in edge plasmas by an order of magnitude for sufficiently large grain and, in agreement with Ref. 2, becomes less important for small grains. Numerical solution of Eq. $(10,11)$ supports our analytic estimates.

Acknowledgements. This work was supported by the DOE Grant DE-FG02-06ER54852 at UCSD. 


\section{References}

[1] S. I. Krasheninnikov, et al., Plasma Phys. Contr. Fusion 53083001 (2011)

[2] B. T. Brown, et al., Phys. Plasmas 21024501 (2014)

[3] K. Saito, et al., J. Nucl. Mater. 363-365 1323 (2007)

[4] S. L. Milora, et al., Nucl. Fusion 35657 (1995)

[5] V. Rozhansky, et al., Plasma Phys. Contr. Fusion 37399 (1995)

[6] L. L. Lengyel, et al., Nucl. Fusion 39791 (1999)

[7] B. V. Kuteev, et al., Sov. J. Plasma Phys. 10675 (1984)

[8] P. B. Parks, et al., Nucl. Fusion 28477 (1988)

[9] D. Kh. Morozov, et al., Nucl. Fusion 44252 (2004)

[10] V. A. Rozhansky and I. Yu. Senichenkov, Plasma Phys. Rep. 31993 (2005)

[11] V. Yu. Sergeev, et al., Plasma Phys. Rep. 32363 (2006)

[12] D. E. Post, J. Nucl. Mater. 220-222 143 (1995)

[13] B. V. Kuteev, et al., Nucl. Fusion 351167 (1995)

[14] T. Pütterich, et al., Nucl. Fusion 50025012 (2010)

[15] Y. Tanaka et al., Phys. Plasmas 14052504 (2014) 


\section{Figure captures}

Fig. 1. Schematic view of both vapor cloud and secondary plasma around the dust grain. Vectors $\mathbf{B}$ and $\mathbf{R}$ are showing the directions of magnetic field and major radius of a tokamak (which coincides with the direction of secondary plasma motion caused by the B-field curvature and grad(B) plasma polarization effects).

Fig. 2. Ablation flux $\Gamma$ as the function of $R_{d}$ for different $T_{i n f}$, found from numerical solution of Eq. $(10,11)$. 


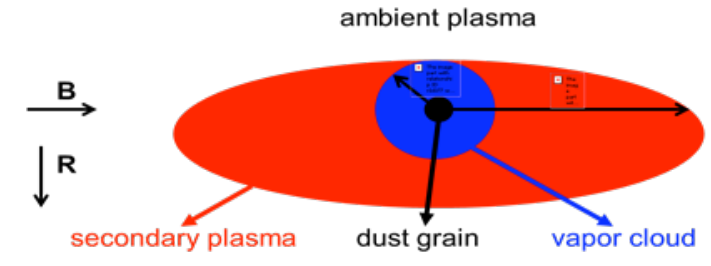

Fig. 1. 


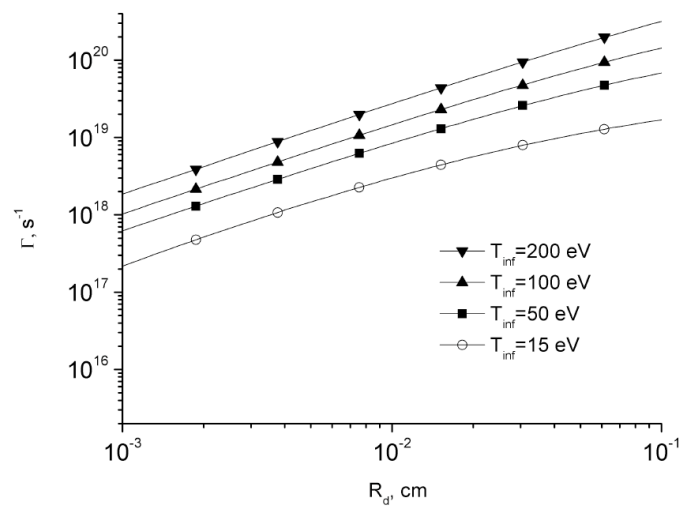

Fig. 2. 